\title{
Los sitios Web de legislación en el Mercosur: un análisis comparativo
}

\author{
Mario Barité \\ Universidad de la República (Uruguay). \\ Profesor titular. \\ E-mail:mabarite@adinet.com.uy
}

María José López-Huertas

Universidad de Granada (España).

Profesora titular.

E-mail:mjlopez@ugr.es

\section{Resumen}

El derecho a la información, que tiene implícito el derecho de acceso a la información, es un pilar fundamental del moderno Estado de Derecho. En el acceso a la información intervienen hoy procesos calificados de organización del conocimiento y de técnicas de recuperación de información, asociadas a las nuevas tecnologías. Las fuentes de información jurídica, en especial en lo que hace a las normas constitucionales y legislativas, deben ser de acceso libre, indiscriminado, gratuito y no restringido, garantizando los derechos fundamentales de los ciudadanos. Es responsabilidad de los Estados facilitar el acceso a esa información, a través de la publicidad de las normas. Internet es un vehículo adecuado para permitir la realización de los principios arriba mencionados del modo más amplio y democrático. En este trabajo se identifican y analizan los sitios oficiales de legislación de cada país integrante del Mercosur. Se caracterizan los sitios en función de parámetros que permitirán cuantificar y calificar el nivel de cumplimiento del principio de publicidad: normas disponibles, período abarcado, formas de acceso, costo, actualización, enlaces, modalidades de recuperación temática, etc. Se realiza un análisis comparativo, se muestran y comentan los resultados y se lleva a cabo una evaluación general.

\section{Palabras clave}

Sitios oficiales de legislación; Mercosur; Análisis comparativo; Normas constitucionales y legislativas; Formas de acceso; Costos; Actualización; Técnicas de recuperación; Evaluación.

\section{Legislative Web sites in Mercosur: a comparative analysis}

\begin{abstract}
The right to information, implicit in the right to information access is one the main basis of the modern State of law. In the access to information, highly qualified processes of knowledge organization and retrieval techniques, associated to new technologies, operate. The sources for legislative information, in special those related to the constitutional and legislative rules, must have a free, no charges access; at the same time, it must be unrestricted and with no discrimination so the citizens fundamental rights are guarantied. The Sates are responsible for easing the access to information by publishing the rules. Internet is an adequate vehicle to put in practice the principles stated above in a more broad and democratic way. In this work, the official web sites on legislation belonging to the countries integrated in Mercosur are analyzed. The web sites are characterized using parameters that allow quantify and qualify the level of fulfilment of the publishing principle: kinds of rules, time period, forms of access, costs, links, updating, ways of information subject retrieval, etc.. A comparative analysis is carried out, the results are shown and discussed, and a general evaluation is done.
\end{abstract}

\section{Keywords}

Official Web sites on legislation; Mercosur; Comparative analysis Constitutional and legislative rules; Forms of access; Costs; Updating; Retrieval techniques; Evaluation.

\section{INTRODUCCIÓN}

En la primera parte de este trabajo, bajo el epígrafe Información y Ciudadanía, se analizan los derechos a la información y al acceso a la información como formas de construcción de la ciudadanía, y la manera en que los procesos de exclusión social afectan a esos derechos. La segunda parte, denominada Información legal en Internet refiere a la naturaleza de la legislación y los documentos legislativos, y a las características documentales que son relevantes para un adecuado acceso a la información por parte de los ciudadanos a través de la Red. En particular, se valoran los atributos con que deben contar los sitios oficiales web de contenido legislativo. En la tercera parte, finalmente, bajo el nombre Los sitios web oficiales de legislación del Mercosur, se desarrolla una investigación comparada entre los sitios de los cuatro países del Área, se presentan los resultados y las conclusiones de la misma.

\section{INFORMACIÓN Y CIUDADANÍA}

\section{El derecho a la información}

El derecho a la información, que tiene implícito el derecho de acceso a la información, es un pilar fundamental del moderno Estado de Derecho. En tanto derecho, está tutelado de modo más o menos expreso en las constituciones modernas de los Estados Iberoamericanos, y queda comprendido dentro de los principios de libre comunicación de ideas, ya que si por regla es libre la emisión de ideas, datos o informaciones, no lo pueden ser menos la búsqueda y el acceso libres a esa información. En el caso de Uruguay, es el artículo 29 de la Constitución el que determina que "es enteramente libre en toda materia la comunicación por palabras, escritos privados o publicados en la prensa, o por cualquier otra forma de divulgación, sin necesidad de censura previa, quedando responsable el autor, y en su caso, el impresor o emisor, con arreglo a la ley por los abusos que cometieren” (Uruguay, 2003).

A este respecto, como expresa Ramón (1998, p. 9), citando en parte a Yglesias (1997, p. 123) se verifica una evolución "desde la libertad de expresión y de informar, hasta llegar a lo que hoy se llama "derecho a la información", ya que "así como la masificación de la información llevó a hablar de 'libertad de información' como un segundo escalón luego de la libertad de 
expresión del pensamiento, el 'derecho a la información' es una reexpresión de ese mismo derecho, esta vez consecuencia de la irrupción de las comunicaciones a través de medios informáticos."

Lo que damos en llamar Sociedad de la Información, se sostiene sobre un vertiginoso desarrollo tecnológico y conceptual en el área de las telecomunicaciones. En este marco, Internet y el correo electrónico son unas de las aplicaciones que han generado mayor repercusión en la literatura y en la vida corriente. No obstante, el principio constitucional que establece la libertad de comunicación como principio y la restricción de la libertad como excepción, se mantiene y hasta se ha extendido en relación con las nuevas tecnologías. Conforme con Barbagelata (1986, p. 61ss.), "el medio técnico que se escoja, aunque tenga protecciones diferentes, aunque tenga repercusiones sociales distintas es una manera idénticamente tutelable de transmitir los pensamientos".

Jardim (1999) apunta que el derecho a la información tiene una triple naturaleza: como derecho civil, como derecho político y como derecho social, y que su ejercicio se limita por las posibilidades de acceso efectivo y capacidad de comprensión de la información disponible con que cuenta un importante sector de la sociedad civil.

El derecho al acceso a la información es una faceta nuclear del derecho de comunicación, y la contracara necesaria del derecho a la información, que presupone que cualquier persona pueda buscar cualquier información, y acceder a la misma en forma libre e irrestricta, salvo razones de Estado (por ejemplo, la información de inteligencia militar), o de empresa (por ejemplo, la información que tiene un costo o aquella catalogada como secreto industrial).

El problema que se plantea es cuán efectivo es el cumplimiento de este derecho en la realidad cuando se habla de información disponible en Internet, debido especialmente a dos razones: a) los niveles de analfabetismo tecnológico o digital existentes en nuestras sociedades; b) las repercusiones que los fenómenos de exclusión social y marginación tienen sobre el acceso a Internet.

En el acceso a la información intervienen hoy procesos calificados de organización del conocimiento y de técnicas de recuperación de información, asociadas a las nuevas tecnologías. La responsabilidad de los gobiernos se centra en la obligación de asegurar el acceso a determinado tipo de información (como la legal o la de servicios a la comunidad), necesaria para garantizar la idea de ciudadanía, por lo que esos gobiernos están obligados al cumplimiento de parámetros mínimos que vuelvan real esa garantía.

\section{LOS CONCEPTOS DE CIUDADANÍA Y DE EXCLUSIÓN SOCIAL}

El derecho de acceso a la información está intrínsecamente relacionado con los conceptos de ciudadanía y de exclusión social, dos aspectos que son básicos para el desarrollo de esta investigación.

\section{a) Concepto de ciudadanía}

Según Targino, citado por Rocha (2000, p. 43), "la ciudadanía es un status concedido a aquellos que son elementos integrales de una comunidad", que conlleva la idea de igualdad de derechos e igualdad ante la ley. No obstante, agrega Rocha, "contrariamente a la idea de igualdad que la ciudadanía sugiere, la estratificación social, que concede el status, es un sistema de desigualdad que se acentúa y se agrava en el sistema capitalista."

Otras definiciones tradicionales, curiosamente, excluyen los derechos sociales como parte integrante de la noción de ciudadanía. Entendemos que esa es una concepción superada, porque es justamente en la posibilidad de que cualquier persona sea ungida efectivamente de todos los derechos sociales, que le corresponden como integrante de una comunidad, que se hace realidad la idea de ciudadanía. Las políticas sociales (de educación, de salud, de vivienda) de los gobiernos más sensibles a esta tendencia, intentan solucionar por este medio las desigualdades propias de toda sociedad. En nuestra perspectiva, el derecho a la información, y el derecho de acceso a la información son derechos sociales, cuyo cumplimiento es imprescindible para asegurar iguales oportunidades de informarse, comunicarse, aprender y crear nuevo conocimiento.

Es recurrente en la literatura especializada, la idea de que la educación (formal e informal) en todos sus niveles y posibilidades, es un elemento esencial para asegurar nuevas formas de ejercicio de la ciudadanía en relación con el acceso a la información. Así, Rocha (2000 p. 43) expresa que "en un cuadro de economía globalizada y de la sociedad organizada a partir del paradigma del conocimiento, el factor educación asume papel fundamental en ese proceso". Morales Campos (2000, p. 17) señala, por su parte, que "una de las respuestas de América Latina ante los desafíos del desarrollo es una educación permeada por la información que debe pasar por un proceso de abstracción, sistematización, experimentación y acción, en busca de la equidad, la calidad y la colaboración". A su juicio, "la información 


\section{Mario Barité / María José López-Huertas}

y la educación son la base de un desarrollo fundado en el pluralismo cultural, la convivencia entre las culturas originarias y populares, por un lado, y las más modernas y globalizadoras por el otro, la multiplicidad étnica al lado de los consensos, la interacción entre lo local, lo regional y lo nacional, entre la sociedad nacional y la sociedad global”.

En ese contexto, parece evidente que se requieren capacidades específicas para acceder a la información en fuentes electrónicas, y para manejar correctamente las herramientas tecnológicas disponibles en Internet, por lo que debe enfatizarse la formación y la capacitación de los ciudadanos en el manejo de nuevas tecnologías. Ding (2000) afirma que el sentimiento general es que el acceso a la información electrónica demanda el conocimiento de diferentes y novedosas técnicas y estrategias de búsqueda, mayores en comparación con las versiones impresas. Aun (1999) lo expresa de forma más dramática cuando manifiesta que las nuevas exigencias educacionales y de capacitación para integrar a los ciudadanos a la sociedad de la información son "un problema para los Estados", y si se nos permite ir un poco más allá, son un problema de Estado, puesto que si este proceso no se cumple, como alerta esta autora "la idea de sociedad de la información en corto tiempo podrá caer en el vacío".

\section{b) Concepto de exclusión social}

Según un documento de OIT/OPS (1999) la exclusión social "corresponde a un estado en el cual el individuo no puede acceder a condiciones de vida que le permitan, al mismo tiempo, satisfacer sus necesidades esenciales (alimentación, educación, salud) y participar en el desarrollo de la sociedad en que vive". Otra definición, algo más extensiva que la primera (disponible en www.iidh.ed.cr), expresa que es la "escasez crónica de oportunidades y de acceso a servicios básicos de calidad, a los mercados laborales y de crédito, a condiciones físicas y de infraestructura adecuada, y al sistema de justicia.”

De estas conceptualizaciones no surge expresamente que las personas, los ciudadanos, requieran hoy la satisfacción de necesidades esenciales de información, tan fundamentales como las otras. Sin embargo, en el actual estado de civilización parece indiscutible que el acceso a la información supone el cumplimiento de una necesidad elemental del ciudadano moderno, ya que a través de esa información resuelve situaciones centrales de su vida vinculadas al trabajo, a la educación, a la alimentación y a la salud. En esta perspectiva más evolucionada se ubican Dutch y Muddiman (2001, p.
183) cuando afirman que "la idea de que la sociedad de la información es, en sí misma, una ruta hacia la eliminación de la exclusión social, es una de las más poderosas y de mayor visibilidad en el debate corriente, porque la exclusión puede ser cultural tanto como material."

Es en este nuevo orden de pensamiento que surgen conceptos nuevos, tales como "infoexclusión”, "apartheid digital", "inforricos" e "infopobres", para caracterizar nuevas formas de marginación, surgidas de la brecha entre las garantías de ciudadanía que se proclaman en el discurso político, y los distintos niveles de exclusión social que provoca el acceso restringido a fuentes electrónicas por razones económicas, culturales o educativas.

En este escenario, aparecen alertas citadas por Aun (1999), quien afirma que "lo que se puede constatar es que en todas las épocas siempre existieron ricos y pobres, excluidos e incluidos; cuando miramos el mundo y las diferencias en el desarrollo de los países, parece lógico que esas diferencias se constaten también en la Sociedad de la Información. Ni la alfabetización ni el hambre han disminuido en porcentaje. Con estos datos básicos, las diferencias en el desarrollo de la Sociedad de la Información pueden ser mayores todavía, debido al grado de exigencias y competencias demandadas para que los países en desarrollo se integren a la nueva era de la información”. Razón de más para profundizar en el estudio de estas cuestiones.

\section{LA INFORMACIÓN LEGAL EN INTERNET}

\section{Pros y contras de la información digital}

Como expresan Dalgleish y Hall (2000, p. 104), Internet "se ha establecido como un medio para la más amplia diseminación de información, al punto que en breve espacio de tiempo la producción de materiales electrónicos ha excedido largamente a la de publicaciones impresas". En la literatura especializada se manifiestan pros y contras de la información digital. A favor, se mencionan:

- Su disponibilidad permanente, sólo afectada por cortes en el servicio.

- La consulta desde cualquier lugar (la casa, el trabajo, la calle, otra ciudad, etc.)

- La inmediatez del acceso.

- La progresiva compatibilización y uniformización de herramientas, de funciones y de íconos identificatorios. 
- La dimensión enorme de información a la que puede accederse, incomparable en relación con su correlato impreso.

- La fiabilidad y credibilidad de los sitios institucionales y gubernamentales, en este último caso, porque contienen información objetiva, como es el caso de la legislación.

Estas ventajas ofrecen un medio de acceso teóricamente libre y democrático, al que podrán sumarse todos los ciudadanos en la medida en que los Estados vayan superando y resolviendo las situaciones de exclusión social y analfabetismo tecnológico.

En contra, se establece que:

- La información en Internet tiene menos estabilidad y puede desaparecer o modificarse sin mayores garantías.

- El acceso a la red implica una inversión que no todo el mundo está en condiciones de hacer, y una capacitación que no todos poseen.

- La actualización de documentos provoca la pérdida de fuentes o versiones originales (Ding, 2000).

- Muchos sitios organizan su información bastante pobremente, y provocan problemas de búsqueda y recuperación. Otros repiten información tomada de otras fuentes sin mayor análisis, y aún en otros casos la fiabilidad y la credibilidad de la información disponible no son mínimamente satisfactorias.

- La información que tiene algún valor económico tiene un precio que no puede pagar todo el mundo, poniendo barreras económicas a datos de interés social.

La información ciudadana puede entenderse, siguiendo a Marcella y Baxter (1999, p. 160; 2000, p. 144ss.), como "la información producida por o acerca de dependencias de gobierno local o nacional, y organizaciones del sector público, que puede ser valiosa para el ciudadano y para su vida cotidiana, o en la participación de los ciudadanos en la formulación de políticas de gobierno."

Los sitios web gubernamentales juegan un importante papel para asegurar el acceso democrático y libre a servicios y contenidos de información ciudadana a través de Internet, disminuyendo los aspectos cuestionados de la información digital. Como manifiesta Da Silveira (2001), los gobiernos deben utilizar Internet como "instrumento de aproximación a la sociedad - por medio de la prestación de servicios y disponibilidad de informaciones-al tiempo de ampliar su capacidad operacional y su área de cobertura.” Los gobiernos tienen, en ese ámbito, una oportunidad excelente para demostrar eficacia administrativa en la aplicación de políticas nacionales de información que articulen conceptual y estratégicamente esos servicios, y para contribuir en beneficio de los ciudadanos en la construcción de lo que Levy (2000) llama inteligencia colectiva, fortaleciendo - de paso - los procesos democráticos más allá de lo formal.

Siguiendo a Da Silveira (2001) "la búsqueda por ofrecer mayor posibilidad de control y transparencia, no se constituye en la única motivación del gobierno para ofrecer servicios e informaciones vía Internet. En tiempos de recursos públicos escasos y una demanda creciente de la sociedad (...) el costo de las transacciones vía Internet, de hecho, es bastante reducido en relación con el costo de aquéllas realizadas por medios tradicionales."

La información legal cae dentro de lo que se entiende como información ciudadana, y es el tipo de información que mejor se ajusta a los principios de acceso libre, gratuito, no restringido e indiscriminado. La información jurídica legal y constitucional es, por naturaleza, pública y en su apropiación social se manifiestan derechos fundamentales de los ciudadanos. Es responsabilidad de los Estados permitir, facilitar y concretar el acceso a esa información en esas condiciones, a través de la publicidad, esto es, la acción de hacer públicas las normas. Esta obligación le ha tocado -durante las décadas en las que el papel ejerció reinadoa los boletines o diarios oficiales impresos, los cuales, sin embargo, han tenido siempre una circulación restringida, compensada por la información -no siempre objetiva o exhaustiva- proporcionada por los medios de comunicación.

Es principio jurídico milenario, establecido en todos los códigos civiles asentados sobre la tradición romana, que Ignorantia legis non excusat (la ignorancia de las leyes no sirve de excusa). La contrapartida a este principio, en las democracias modernas, se da por esa obligación estatal de facilitar y asegurar el conocimiento de las leyes, dándoles la más amplia e irrestricta publicidad. La revolución tecnológica no sólo aportó nuevas herramientas digitales sino la posibilidad de volver realidad, de un modo más claro y transparente, esa obligación de dar acceso libre a la información legal a todos los ciudadanos. Por ese motivo, el Estado debe asumir nuevas obligaciones en el marco de la Sociedad de la Información, para garantizar la idea de ciudadanía. Ese esfuerzo se plasma en la presentación de sitios web especialmente diseñados para exhibir información legal. 


\section{Mario Barité / María José López-Huertas}

El Estado no puede desasirse de obligaciones esenciales para ese cumplimiento, sobre todo en lo que hace a campañas de alfabetización informática y de democratización del acceso a las computadoras.

\section{LA LEGISLACIÓN}

Los sitios oficiales de legislación deben contener, en primer lugar, legislación. Pero, ¿qué se entiende por legislación? Conforme con Guimaraes (Barité y Guimaraes, 1999, p. 13) "la legislación corresponde, materialmente, al conjunto de los actos legislativos y normativos emanados de autoridad competente". Como apunta Barité (1999, p. 22), "importa tanto considerar a la información que corresponde a la legislación en vigor en un país (el llamado derecho positivo vigente), como a aquella que refiere a normas de cualquier estirpe que han sido derogadas, en virtud de que unas y otras son potencialmente requeridas por los usuarios." A efectos de identificar la legislación no válida, el usuario debe poder distinguir claramente entre normas vigentes y normas derogadas, contando con el auxilio de las referencias que indiquen claramente los casos de modificación de textos legales, y las fechas de vigencia en cada caso. En los sitios gubernamentales este principio debe cumplirse a satisfacción, y es uno de los puntos evaluados en el presente estudio.

Los procesos de modificación de las normasextremadamente comunes en Derecho- exigen un tratamiento exhaustivo y detallado, que se expresa mediante un sistema de referencia de coordinación temporal, el que encuentra en los vínculos hipertextuales una herramienta especialmente idónea. Los tipos de modificación de las leyes son, como es sabido, derogación, sustitución y agregación.

La actualización de este sistema de correlaciones es esencial para el conocimiento y el trabajo especializado de los distintos interesados (abogados, jurisconsultos, notarios, etc.), quienes incluso pueden sufrir o provocar serias consecuencias económicas, profesionales y legales de acudir para sus prácticas profesionales concretas, a normativas no vigentes.

La legislación tiene unas características muy peculiares derivadas de su fuerte complejidad interna (Barité, 1999). Por esta razón, requiere una organización mixta que debe contemplar al menos cinco aspectos para una búsqueda consolidada en Internet:

- Jerárquico. El primer elemento de identificación debe ser el rango legal de la norma, y las relaciones que tiene con otras normas (decretos, resoluciones, etc.).
- Numérico. La ordenación de leyes por su número es una organización tradicional y no sistemática de las leyes que otorga una ventaja indiscutible de ofrecer un punto de acceso que asegura una entrada exclusiva y excluyente. Tiene, no obstante, el inconveniente de que ese número debe conocerse previamente.

- Cronológico. Permite establecer los extremos de vigencia de la norma, y sus variaciones en el tiempo. El orden cronológico de las leyes se yuxtapone al numérico en la mayoría de los ordenamientos jurídicos.

- Temático. Permite obtener el acceso por tópicos. Es tendencia preferida en los sitios web de legislación establecer, a diferencia de muchos boletines o diarios oficiales, descriptores temáticos para identificar cada texto legal, e incluso nombres de personas y de lugares mencionados en las leyes.

- Acceso por lenguaje natural. El acceso por lenguaje natural puede hacerse a lo largo de todo el texto de la norma, y también por su título (por ejemplo, "Ley de Seguridad Ciudadana”, o "Ley de Violencia Doméstica”). Esta función permite complementar el acceso formalizado por descriptores, ya que incorpora la totalidad de las palabras significativas presentes en los textos legales, si bien agrega el problema de la polisemia o la ambigüedad.

Para ciertas necesidades o requerimientos de información, es preciso, incluso, permitir la combinación de aspectos, como cuando se requiere recuperar todas las normas relativas a una temática determinada, sin importar su rango jerárquico.

\section{Los sitios web oficiales de legislación}

Son los encargados de alojar y difundir la información legislativa y deben cumplir con una serie de condiciones, cometidos y objetivos de naturaleza formal, informativa y ergonómica -algunos de ellos comunes a todos los sitios de Internet- como forma de garantizar más firmemente la idea de ciudadanía en Internet. Formalmente, los sitios exigen desarrollarse a partir de una arquitectura de información que utilice patrones y herramientas ya difundidos universalmente en Internet (por ejemplo, el dispositivo establecido para indicar un vínculo hipertextual). Desde el punto de vista informativo, los sitios requieren el máximo aprovechamiento y la mayor versatilidad de las herramientas para acceder a la información desde muchos puntos de partida. Respecto a la visión ergonómica, señalan Barboza, Nunes y Sena (2000, 123) que "según la ISO 9241, la interacción entre el usuario y el sistema de recuperación de la información 
alcanzará su objetivo específico, utilizando principios ergonómicos generales, cuando se proyecte una interfase con terminal de video: adecuada para la tarea, autodescriptiva, controlable, estar conforme con las expectativas de los usuarios, propiciar el aprendizaje" entre otros. Estos autores afirman $(2000,118)$ que compete "a un equipo multidisciplinario la responsabilidad de llevar [el sitio] a la excelencia en su calidad, favoreciendo la colecta, reproducción, indización y diseminación de informaciones, de forma segura, para un público extremadamente diverso".

A continuación se desarrollan en forma sumaria algunos caracteres que entendemos relevantes para los sitios de legislación:

- Deben estar a cargo del Parlamento o Congreso del país, por razones pragmáticas y simbólicas.

- Deben contener información legal objetiva, consistente en la transcripción literal y oficial de los textos legales tal como han sido promulgados.

- Los códigos y los tratados internacionales firmados y/ o ratificados por el país deben contar con entradas directas, aunque tengan rango de ley.

- Deben contener sumarios o encabezados de las leyes, que faciliten búsquedas intermedias o sumarias de información.

- La información debe ser fiable, confiable por su fidelidad, en razón de que la responsabilidad institucional recae directamente en el Estado.

- Deben asegurarse mecanismos de actualización periódica de la legislación, y el avance retrospectivo (o avance hacia atrás) en la cobertura legal digital hasta su total incorporación. De este modo, además de brindar la información, la misma actúa, en palabras de Guimaraes (1999, p. 12), "como soporte de la memoria de la sociedad, por medio de la actuación del Estado."

- Deben ser gratuitos, y ofrecer un acceso directo, sin contraseñas, al texto integral de las leyes.

- Deben constituirse en nodos para la concreción de portales cooperativos, en donde se asocie información de diferentes dependencias del Estado, evitando la búsqueda aislada del usuario, para su posterior consolidación.

Por otra parte, es necesario considerar los siguientes aspectos para facilitar la comunicación sitio-usuario:
- La continuidad y la permanencia del sitio.

- La definición de perfiles de usuario, conforme con los servicios de información ofrecidos (Fleming, 1998), para ajustar unos a otros.

- La concepción del sitio como un escenario activo dentro del cual navegan personas con distintos intereses y necesidades particulares (Nielsen, 2000).

- La utilización de lenguaje preciso, en gramática y sintaxis correcta (Da Silveira, 2001).

- La presencia de interfases, y herramientas de búsqueda y ayuda que permitan la mejor satisfacción de los usuarios.

- La utilización de elementos didácticos que contribuyan al proceso individual de conocimiento de herramientas y técnicas de búsqueda.

- Características técnicas que aseguren el acceso a poseedores de computadoras de anteriores generaciones. (Nielsen, 2000).

- La existencia de modalidades para la comunicación del usuario con los responsables del sitio, mediante correo electrónico, buzón de sugerencias y herramientas similares, que permitan la retroalimentación sobre opiniones y sugerencias de ciudadanos.

- Facilidades para obtener copias de textos legales.

- La existencia y control periódico de links con páginas externas de legislación y de prensa, parlamentos, etc., favoreciendo formas de link popularity (Scott, 2003, p. 625).

- La aplicación de criterios de usabilidad y accesibilidad que cobran cada vez mayor importancia en la gestión de Internet (Barboza, Nunes y Sena, 2000, p. 119; Hassan, 2002; Hassan y Martín, 2003; Henry 2002 y 2003).

\section{LOS SITIOS WEB OFICIALES DE LEGISLACIÓN DEL MERCOSUR}

\section{Objetivos de la investigación}

El objetivo general de la presente investigación, que se sustenta en la filosofía arriba expuesta, es analizar y comparar los sitios web de legislación de los países del Mercosur, a efectos de dar pautas para la implantación futura de un modelo común en lo relativo a arquitectura de información, interfases, información disponible, herramientas y enlaces, favoreciendo una conjunción de sus mejores elementos particulares, en términos de presentación de datos, usabilidad y accesibilidad. 


\section{Mario Barité / María José López-Huertas}

Como objetivos específicos se señalan los siguientes:

- Describir la realidad actual de los sitios web de legislación en el Mercosur.

- Identificar los elementos necesarios para promover una unificación en la arquitectura de los sitios de los países del Mercosur, y el enlace de unos con otros.

- Consolidar la noción de ciudadanía y sus formas de garantía, evaluando aspectos conceptuales y políticos del acceso a la legislación en Internet.

- Contribuir a socializar la necesidad de disminuir la exclusión social en general, y la infoexclusión en particular.

- Valorar la responsabilidad institucional de los respectivos Parlamentos o Congresos

\section{Material y métodos}

Para llevar a cabo el estudio, se utilizó como material de trabajo un corpus digital que estuvo formado por los sitios web oficiales de legislación de los países del Mercosur:

- ARGENTINA: www.hcdn.gov.ar

<http://www.hcdn.gov.ar/>

- BRASIL: www.senado.gov.br

<http://www.senado.gov.br/>

- PARAGUAY: www.senado.gov.py

<http://www.senado.gov.py/>

- URUGUAY: www.parlamento.gub.uy

<http://www.parlamento.gub.uy/>

\section{Metodología utilizada}

La investigación se realizó desde la perspectiva de un ciudadano común, es decir que se analizaron los sitios simulando las búsquedas que podría realizar cualquier persona en cualquier momento desde cualquier punto del planeta. Los análisis se realizaron en los meses de setiembre y octubre de 2003.

El método de análisis utilizado siguió las etapas que se detallan a continuación:

1. Análisis particular de la estructura normativa de legislación de cada país del Mercosur, en función de su naturaleza político-adminitrativa (naturaleza federativa o no de los cuatro países, etc.).

2. Identificación y relevamiento de los datos en los sitios.
3. Determinación de los parámetros de análisis considerando tanto aspectos conceptuales asociados con la garantía de la ciudadanía. Para establecer los parámetros de análisis, se tomaron en consideración indicadores vinculados conceptualmente con la idea de garantizar la ciudadanía por parte del Estado en Internet. Estos parámetros se dividieron en: I) cobertura; II) accesibilidad; III) interfases en términos de enlaces; IV) herramientas.

I. PARÁMETROS DE COBERTURA. Dentro de este apartado se consideraron los siguientes indicadores:

- Normas disponibles. Si bien el estudio está centrado en la legislación, se tuvo en cuenta el acceso independiente a los Códigos (que tienen rango de ley), a la Constitución, a decretos y otras normas administrativas, o de naturaleza provincial o local.

- Cantidad de leyes disponibles. Refiere al número de leyes efectivamente accesibles.

- Periodo. Se consignan los años de legislación que están disponibles.

- Actualización. Se identifican los siguientes elementos: 1. Actualización retrospectiva, en razón de que muchos sitios todavía no han completado la versión digital de toda su legislación. 2. Actualización prospectiva. También se indica la periodicidad de las actualizaciones si se presenta el dato.

II. PARÁMETROS DE ACCESIBILIDAD. Es un concepto importante a los efectos de la evaluación de sitios web. Se asocia en ciertos contextos al conjunto de facilidades establecidas para que personas discapacitadas puedan acceder a los contenidos del sitio. Este concepto se ha ampliado para referirse a la "posibilidad de que un producto o servicio web pueda ser accedido y usado por el mayor número posible de personas, indiferentemente de las limitaciones propias del individuo o de las derivadas del contexto de uso" (Hassan y Martín, 2003). Dentro de este apartado se consideran los siguientes indicadores:

- Puntos de acceso: Se consideran como tales los accesos numéricos, cronológicos, por descriptores, por lenguaje natural, por tipo de norma (en el caso de sitios que incluyen normativa de diferente jerarquía) y por fuente de la norma (es decir, la indicación del órgano de origen o de la publicación original).

- Acceso a texto completo.

- Acceso a sumario o al encabezamiento del texto. 
- Costo: Se determina si el acceso para el usuario es libre o tiene un precio.

\section{PARÁMETROS DE INTERFASE (ENLACES)}

- Enlaces internos: enlaces a historia de la norma, a normas relacionadas, a otras normas mencionadas en el texto de una ley, y enlaces a normas derogadas o modificadas.

- Enlaces externos: se discrimina entre enlaces a sitios oficiales del mismo país, a sitios no oficiales nacionales o internacionales, y en particular, enlaces a los otros sitios oficiales de legislación de países del Mercosur.

- Correo electrónico para usuarios. Refiere a la existencia de un espacio destinado a permitir la comunicación directa del ciudadano con el Congreso o Parlamento.

\section{HERRAMIENTAS}

- Número de documentos por pantalla.

- Sumario.

- Búsquedas combinadas por distintos elementos.

- Búsquedas avanzadas utilizando operadores lógicos.

- FAQs. La sigla corresponde a "frequently asked questions", es decir, las preguntas que más frecuentemente recibe un sitio o un servicio de información.

- Hipervínculos.

- Ayudas.

\section{RESULTADOS}

Una vez analizados los materiales seleccionados, se obtuvieron los siguientes resultados:

\section{PARÁMETROS DE COBERTURA}

- Las Normas disponibles en los sitios consultados se muestran en la tabla 1.

TABLA 1

Normas disponibles en los sitios consultados

\begin{tabular}{l|c|c|c|c}
\hline & Argentina & Brasil & Paraguay & Uruguay \\
\hline Constitución & Sí & Sí & Sí & Sí \\
Tratados & No & No & No & No \\
Códigos & No & No & No (P1) & No \\
Leyes & Sí & Sí & Sí & Sí \\
Decretos & Sí & Sí & No & No \\
Otras normas & Sí & Sí & Sí & No \\
\hline
\end{tabular}

(P1) Es posible acceder a los Códigos indirectamente, escribiendo la palabra "Códigos" en una ventana de búsqueda que ofrece el sitio.

- Otros parámetros de cobertura como son la cantidad de leyes, el período cubierto y la actualización se muestran en la tabla 2.

\section{TABLA 2}

Otros parámetros de cobertura

\begin{tabular}{l|c|c|c|c}
\hline & Argentina & Brasil & Paraguay & Uruguay \\
\hline Cantidad leyes & 2.741 & No dice & No dice & 8.195 \\
Período & $10 / 12 / 83$ a & No dice & $1998-2002$ & $13 / 09 / 35$ a \\
& $1 \% / 10 / 03$ & & & $16 / 10 / 03$ \\
Actualización & No dice & No dice & No dice & No dice \\
\hline
\end{tabular}

\section{PARÁMETROS DE ACCESIBILIDAD}

- Los puntos de acceso a la legislación en los distintos sitios consultados se muestran en la tabla 3 .

TABLA 3

Puntos de acceso

\begin{tabular}{l|c|c|c|c}
\hline & Argentina & Brasil & Paraguay & Uruguay \\
\hline Numérico & Sí & Sí & No (P1) & Sí \\
Cronológico & Sí & Sí & Sí & No \\
Descriptores & Sí & Sí & No & Sí (U1) \\
Lenguaje natural & Sí & Sí & Sí & Sí (U2) \\
Tipo norma & Sí & Sí & No & No \\
Fuente de la norma & No & Sí (B1) & No & Sí \\
\hline
\end{tabular}

(B1) El sitio muestra dónde fue publicada la norma por primera vez.

(P1) Se ofrece un índice de materias como base para el acceso, pero no está operativo.

(U1) Agrega ventana con descriptores desarrollados.

(U2) Incluye autoridades patronímicas y de nombre geográficos, tal como aparecen en la legislación.

- Otros parámetros de accesibilidad considerados, como el acceso a texto completo, acceso a sumario y costo se muestran en la tabla 4.

TABLA 4

Otros parámetros de accesibilidad

\begin{tabular}{l|c|c|c|c}
\hline & Argentina & Brasil & Paraguay & Uruguay \\
\hline Acceso a texto & & & & \\
completo & No (A1) & Sí & Sí & Sí \\
Acceso a sumario & Sí & Sí & No (P1) & Sí (U1) \\
Costo & No & No & No & No \\
\hline
\end{tabular}


(A1) No se accede al texto completo de las leyes. Algunas bases sólo están disponibles para usuarios de intranet.

(P1) Los sumarios sólo son visibles bajo el índice de materias.

(U1) Se accede al texto que encabeza la norma (las primeras frases de la misma) y no a un sumario.

\section{PARÁMETROS DE INTERFASE EN TÉRMINOS DE ENLACES}

- Los Enlaces internos en los sitios web consultados se muestran en la tabla 5 .

TABLA 5

Enlaces internos

\begin{tabular}{|c|c|c|c|c|}
\hline & Argentina & Brasil & Paraguay & Uruguay \\
\hline $\begin{array}{l}\text { A historia de la } \\
\text { norma }\end{array}$ & No & No & No & Sí \\
\hline $\begin{array}{l}\text { A normas } \\
\text { relacionadas }\end{array}$ & Sí (A1) & Sí & Sí & Sí \\
\hline $\begin{array}{l}\text { A normas } \\
\text { encionadas en la ley }\end{array}$ & No & $\mathrm{Si}$ & No & Sí \\
\hline $\begin{array}{l}\text { A normas } \\
\text { modificadas }\end{array}$ & Sí (A1) & Sí & No & Sí \\
\hline
\end{tabular}

(A1) Enlace a modificaciones posteriores al texto de la ley. También establece vínculos con reglamentaciones y otros textos de menor jerarquía asociados a una ley.

- Los Enlaces externos se indican en la tabla 6.

TABLA 6

Enlaces externos

\begin{tabular}{l|c|c|c|c}
\hline & Argentina & Brasil & Paraguay & Uruguay \\
\hline $\begin{array}{l}\text { Correo electrónico } \\
\text { A sitios oficiales }\end{array}$ & No (A1) & Sí & & Sí \\
mismo país & Sí & Sí (B1) & Sí & Sí \\
$\begin{array}{l}\text { A sitios no oficiales } \\
\text { Enlaces a sitios } \\
\text { legislación Mercosur }\end{array}$ & Sí & Sí (B2) & No & Sí \\
\hline
\end{tabular}

(A1) No existe un correo electrónico propiamente dicho, pero el sitio permite que los usuarios se registren para recibir información o para realizar consultas.

(B1) Ofrece enlaces a 27 tipos diferentes de normas de rango federal, estadual, constitucional, legal, decretos leyes, decretos, resoluciones y actos de diverso orden.

(B2) Enlaces a prensa digital, a agencias de noticias, etc.

\section{IV) HERRAMIENTAS}

\begin{tabular}{l|c|c|c|c}
\hline & Argentina & Brasil & Paraguay & Uruguay \\
\hline$N^{\circ}$ doc. X Pantalla & No & Sí & No & Sí \\
Búsquedas combinadas & Sí & Sí & No & Sí \\
Búsquedas avanzadas & Sí & Sí & No & Sí \\
FAQ's & No & No & Si (P1) & Si \\
Hipervínculos & Sí & No & Sí & Sí (U1) \\
Ayuda & No & Sí (B1) & Sí & Sí \\
\hline
\end{tabular}

(B1) Presentación de ejemplos e instrucciones de búsqueda en lenguaje sencillo. Agrega explicación de operadores lógicos.

(P1) Cuenta con un enlace a las leyes de uso más frecuente.

(U1) Los hipervínculos se establecen en las remisiones entre leyes, entre las leyes y la Constitución, y entre la ley y la historia de su trámite parlamentario.

\section{CONCLUSIONES}

Parece importante en lo simbólico que los sitios dependan del Parlamento o Congreso Nacional.

En cuanto a los parámetros de cobertura las conclusiones son las siguientes:

- La cantidad de leyes disponibles es baja, considerada tanto porcentualmente como en números absolutos. Esta disponibilidad parcial atenta contra la excelencia del servicio y contra el cumplimiento del libre acceso a la información en Internet.

- Se observa como problema la ausencia de accesos directos a los códigos, y la imposibilidad de acceder a los tratados internacionales suscritos y/o ratificados por los respectivos países.

- Se verifican lagunas grandes en la cobertura retrospectiva en los cuatro países. Sólo Uruguay ha logrado incorporar legislación con una antigüedad mayor a 20 años.

- Los sitios no indican la periodicidad ni las características de las actualizaciones.

En cuanto a los parámetros de accesibilidad, los puntos de acceso fundamentales parecen estar cubiertos en forma aceptable:

- Tres de los cuatro países ofrecen acceso libre al texto completo de las leyes. Curiosamente, Argentina no da esta posibilidad, ni en forma gratuita ni en forma paga, constituyendo una limitación importante a la garantía de ciudadanía. 
- Los cuatros países cumplen con el principio de gratuidad de acceso a la información legislativa.

Respecto a los parámetros de interfase:

- Sorprende la escasa utilización de vínculos hipertextuales para conectar normas relacionadas, para enlazar normas mencionadas en el texto de una ley, o para establecer la relación con normas derogadas o modificadas.

- Es notable la carencia de enlaces a la historia parlamentaria de la norma, salvo en el caso de Uruguay.

- Hay una cobertura satisfactoria de enlaces externos. Sin embargo constituye un déficit extraordinario la ausencia de enlaces directos y claramente distinguibles, entre los sitios oficiales de legislación de países del Mercosur. En ninguno de los cuatro sitios existe una preocupación por relacionar en forma específica el sitio con los otros, por lo cual la idea de integración en esta materia ha quedado sólo en el papel hasta el presente.

Las herramientas utilizadas, no están unificadas. Existe una gran dispersión de las mismas, y en general se verifica que los diseños son diferentes y poco compatibles en la actualidad. Hay carencia de ayudas más efectivas en algunos de los sitios para favorecer la navegación.

Si bien puede ser materia opinable, los autores evalúan que los sitios de Brasil y Uruguay son muy amigables, que el sitio de Paraguay reúne características satisfactorias, aunque muchas de las herramientas e interfases previstas no están operativas, y que el sitio de Argentina necesita una redefinición de su diseño.

Cabe manifestar, finalmente, que el panorama que ofrecen los sitios de legislación de países del Mercosur es, a la vez, alentador y preocupante. Alentador porque parece existir la intención, en los cuatro países, de desarrollar y fortalecer estos sitios bajo un enfoque de garantía de la noción de ciudadanía. Implícitamente se aboga por la no exclusión social o no infoexclusión, al menos como aspiración. Preocupante, porque los sitios muestran lagunas, poca compatibilidad, y dificultades evidentes para favorecer la búsqueda y para lograr una cobertura retrospectiva satisfactoria.

A partir de estas conclusiones, nos hemos permitido enumerar una serie de recomendaciones que podrían ayudar a la mejora de los sitios web legislativos consultados, favoreciendo la creación de sitios de excelencia, que cuenten con diseños similares, compatibles y comunicados entre sí:
1. Tres de los cuatro Estados tienen una política de acceso libre al texto integral de la legislación, y es una tendencia que tendría que incorporar prontamente al otro país (Argentina).

2. Parece adecuado al cumplimiento efectivo de la integración regional, que los cuatro sitios establezcan enlaces recíprocos, como una forma de reconocimiento y cooperación elementales.

3. Se recomienda una coordinación entre los equipos responsables de los cuatro sitios, a efectos de unificar o compatibilizar progresivamente la arquitectura y las herramientas de búsqueda.

\section{Artigo recebido em 01-06-2004 e aceito para publicação de 18 a $21 / 10 / 2004$.}

\section{REFERÊNCIAS}

AUN, Marta Pinheiro. A construção de políticas nacional e supranacional de informação: desafio para os estados nacionais e blocos regionais. Ciência da Informação, Brasília, v. 28, n. 2, 1999.

BARbagelatA, Aníbal. Derechos fundamentales. Montevideo : Fundación de Cultura Universitaria, 1986.

BARBOSA, E. M.F; NUNES, E. M. A.; SENA, N. K. Web sites governamentais: uma esplanada a parte. Ciência da Informação, Brasília, v. 29, n. 1, p. 118-125, jan./abr. 2000.

BARITÉ, Mario; GUIMARAES, José Augusto Chaves. Guia metodológica para el acceso, el análisis y la organización temática de documentos jurídicos. Montevideo : Universidad de la República, Comisión Central de Educación Permanente, 1999. 159 p.

DALGLEISH, Andrew ; HALL, Robert. Uses and perceptions of the world wide web in an information-seeking environment. Journal of Librarianship and Information Science, v. 32, n. 3, p. 104-116, Sept. 2000.

DING, Choo Ming. Access to digital information: some breakthroughs and obstacles. Journal of Librarianship and Information Science, v. 32, 1, p. 26-32, Mar. 2000

DUTCH, Martin; MUDDIMAN, Dave. The public library, social exclusion and the information society in the United Kingdom. Libri, v. 51, n. 4, p. 183-194, Dec. 2001.

FLEMING, J. Web navigation: designing the user experience. [S. 1.] : O'Reilly, 1998.

HASSAN MONTERO, Yusef; MARTÍN FERNÁNDEZ, Francisco. ¿Qué es la accesibilidad web? Disponível em: 〈www.nosolousabilidad.com>.

$$
\text { Introducción a la usabilidad. Disponível em: }
$$

<www.nosolousabilidad.com>.

HENRY, Shawn Lawton. Understanding web accessibility. In: CONSTRUCTING accessible web sites. Disponível em: <http:// www.macromedia.com/macromedia/accessibility/pub/ acc_sites_chap01.pdf>.

Another ability: accessibility primer for usability specialists. In: USABILITY PROFESSIONALS' ASSOCIANTION CONFERENCE, 2003. Disponível em: <http://www.upassoc.org/ conf2003/call/downloads/01-Another-Ability.pdf>. 


\section{Mario Barité / María José López-Huertas}

INTERNATIONAL ORGANIZATION FOR STANDARDIZATION. Ergonomics requirements for office work with visual display terminals. Génova, 1996-1998.

JARDIM, J. M. Entre o vital e o virtual: o acesso aos arquivos públicos no Brasil. [S. 1.: s. n.], 1999. 14 p.

LÉVY, Pierre. Entrevista. Correio Braziliense, Brasília, 4 jun. 2000.

LUCAS, Wendy; TOPI, Heikki. Form and function: the impact of query term and operator usage on Web search results. ASIST, v. 53, n. 2, p. 95-108, 2002.

MARCELLA, Rita; BAXTER, Graeme. Information need, information seeking behaviour and participation, with special reference to needs related to citizenship: results of a national survey. Journal of Documentation, v. 56, n. 2, p. 136-160, Mar. 2000.

The information needs and the information seeking behaviour of a national sample ot the population in the United Kingdom, with special reference to needs related to citizenship. Journal of Documentation, v. 55, n. 2, p. 159-183, Mar. 1999.

ORALES CAMPOS, Estela. El fin de siglo, la globalización y la infodiversidad. Investigación Bibliotecológica, v. 14, n. 28, p. 11-33, ene./jun. 2000

NIELSEN, Jakob. Beyond accessibility: treating people with disabilities as people. Disponível em: <http://www.useit.com/alertbox/ 20011111.html>.
Designing web usability. Indianápolis : New Riders, 2000.

ORGANIZAÇÃO INTERNACIONAL DO TRABALHO/ ORGANIZAC̄̃̃O PAN-AMERICANA DA SAÚDE. Reunión tripartita OIT/OPS: extensión de la protección social en salud a los grupos excluidos en América Latina y el Caribe. Disponível em: <www.lachsr.org/spanish/00002360.htm>.

RAMÓN, María Laura. Algunos aspectos de la libertad de información en la peculiar era de la informática. In: CONGRESO IBEROAMERICANO DE DERECHO E INFORMÁTICA, 6., 1998, Montevideo. [S. 1. : s. n.], 1998. p. 9-17.

ROCHA, M. P. C. A questão cidadania na sociedade da informação. Ciência da Informação, Brasília, v. 29, n. 1, p. 40-45, jan./abr. 2000.

SCOTT, John. How friendly are UK government websites? In: TENDENCIAS de investigación en organización del conocimiento. Salamanca Universidad de Salamanca, 2003. p. 621-629.

SILVEIRA, H. F. R. da. Internet, governo e cidadania. Ciência da Informação, Brasília, v. 30, n. 2, p. 80-90, maio/ago. 2001.

URUGUAY. Constitución (2003). Disponível em: 〈www.parlamento.gub.uy>.

YGLESIAS PEROLO, Arturo. Derecho de las cosas. Montevideo : Fundación de Cultura Universitaria, 1997. 